\title{
Low Dose Curcumin Administered in Hyaluronic Acid-Based Nanocapsules Induces Hypotensive Effect in Hypertensive Rats
}

This article was published in the following Dove Press journal: International Journal of Nanomedicine

\author{
Izabela Czyzynska- \\ Cichon iD 1 ** \\ Małgorzata Janik-Hazuka (iD ${ }^{2, *}$ \\ Joanna Szafraniec-Szczęsny (iD) ${ }^{2,3}$ \\ Krzysztof Jasinski iD ${ }^{4}$ \\ Władysław P Węglarz (iD ${ }^{4}$ \\ Szczepan Zapotoczny (iD ${ }^{2}$ \\ Stefan Chlopicki (iD) ${ }^{1,5}$ \\ 'Jagiellonian University, Jagiellonian \\ Centre for Experimental Therapeutics \\ (JCET), Krakow, 30-348, Poland; \\ ${ }^{2}$ Jagiellonian University, Faculty of \\ Chemistry, Krakow, 30-387, Poland; \\ ${ }^{3}$ Jagiellonian University Medical College, \\ Faculty of Pharmacy, Department of \\ Pharmaceutical Technology and \\ Biopharmaceutics, Krakow, 30-688, \\ Poland; ${ }^{4}$ Institute of Nuclear Physics \\ Polish Academy of Sciences, Department \\ of Magnetic Resonance Imaging, Krakow, \\ 31-342, Poland; Jagiellonian University \\ Medical College, Faculty of Medicine, \\ Department of Pharmacology, Krakow, \\ $3|-53|$, Poland
}

*These authors contributed equally to this work
Background: Vascular drug delivery becomes a promising direction in the development of novel therapeutic strategies in the treatment of cardiovascular pathologies, such as hypertension. However, targeted delivery of hydrophobic substances, with poor bioavailability, remains a challenge. Here, we described the hypotensive effects of a low dose of curcumin delivered to the vascular wall using hyaluronic acid-based nanocapsules.

Methods: The group of hypertensive TGR(m-Ren2)27 rats, was administrated respectively with the vehicle, curcumin solution or curcumin delivered using hyaluronic acid-based nanocapsules (HyC12-Cur), for 7 days each, maintaining the wash-out period between treatments. Arterial blood pressure (systolic - SBP, diastolic - DBP) and heart rate (HR) were monitored continuously using a telemetry system (Data Science International), and Mean Arterial Pressure (MAP) was calculated from SBP and DBP.

Results: In hypertensive rats, a low dose of curcumin $(4.5 \mathrm{mg} / \mathrm{kg})$ administrated in HyC12-Cur for 7 days resulted in a gradual inhibition of SBP, DBP and MAP increase without an effect on HR. At the end of HyC12-Cur - based treatment changes in SBP, DBP and MAP amounted to $-2.0 \pm 0.8$ $\mathrm{mmHg},-3.9 \pm 0.7 \mathrm{mmHg}$ and $-3.3 \pm 0.7 \mathrm{mmHg}$, respectively. In contrast, the administration of a curcumin solution $(4.5 \mathrm{mg} / \mathrm{kg})$ did not result in a significant hypotensive effect and the animals constantly developed hypertension. Vascular delivery of capsules with curcumin was confirmed using newly developed fluorine-rich nanocapsules (HyFC10-PFOB) with a shell based on a HA derivative and similar size as $\mathrm{HyC} 12-\mathrm{Cur}$. HyFC10-PFOB gave fluorine signals in rat aortas analyzed ex vivo with a ${ }^{19} \mathrm{~F}$ NMR technique after a single intragastric administration.

Conclusion: These results suggest that nanocapsules based on hyaluronic acid, the ubiquitous glycosaminoglycan of the extracellular matrix and an integral part of endothelial glycocalyx, may represent a suitable approach to deliver hydrophobic, poorly bioavailable compounds, to the vascular wall.

Keywords: nanocapsules, hyaluronic acid, ${ }^{19} \mathrm{~F}$ NMR, curcumin, hypertension, targeted vascular delivery

\section{Introduction}

The main goal of any therapy is to achieve maximum results of the treatment while avoiding side effects. However, most of the drugs, used against certain pathologies, including cardiovascular diseases, do not have the affinity to desired targets in the body. ${ }^{1}$ Moreover, over $40 \%$ of approved pharmaceuticals and nearly $90 \%$ of the newly synthesized chemicals are sparingly soluble in an aqueous environment, and even they have therapeutic potential, they are rejected at the early stage of
Correspondence: Stefan Chlopicki; Szczepan Zapotoczny Email stefan.chlopicki@jcet.eu; zapotocz@chemia.uj.edu.pl
International Journal of Nanomedicine 2021:16 1377-1390

1377

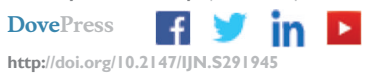


research. ${ }^{2}$ Furthermore, poorly water-soluble or nonselective drugs often require high doses or prolonged infusion to reach therapeutic plasma concentrations, which might eventually occur toxic for organisms. ${ }^{3,4}$

Up to date, many physical, chemical and miscellaneous methods have been revealed to improve the properties of therapeutic agents. ${ }^{4}$ The most promising and developing concept is the use of drug delivery systems (DDSs), especially the ones of the nanometer size, ie liposomes, micelles, nanoparticles, etc. ${ }^{5}$ Enclosing active agents in DDS, enables the administration of hydrophobic or unstable drugs and preferential delivery of non-selective compounds due to the shell properties or DDS architecture. ${ }^{3,6,7}$ Additionally, nanoencapsulation prolongs drug clearance and protects pharmaceuticals from deactivation or degradation, allowing for their controlled and sustained release. $^{7,8}$

A high worldwide prevalence of cardiovascular diseases has contributed to a rapid increase in interest in novel therapeutic strategies using DDSs, which are targeted to the vascular wall. Most of those platforms are based on organic nanocarriers such as liposomes, which surface was additionally modified with specific ligands or coatings (eg monoclonal antibodies, peptides, growth factors, or polymers) enhancing their affinity to the endothelium. $^{9-11}$ For example, liposomes conjugated to anti-ICAM-1 or anti-PECAM antibodies were capable of delivering antioxidant or anti-thrombotic enzymes to the vessel wall in models of oxidative stress, ${ }^{12-14}$ while additional covering with polyethylene glycol (PEG), prolonged their lifetime in the circulation. ${ }^{15,16}$ Moreover, peptidecoated or positively charged liposomes, as well as nanoparticles, were also shown to be effective in vasculature targeting of hydrophobic anti-cancer drugs and natural chemotherapeutics. ${ }^{11,17,18}$ Considering these facts, encapsulation of non-specific and lipophilic molecules like curcumin, lycopene, resveratrol, or quercetin, which are known for their multiple health benefits including cardioand vascular protection, might be useful in targeted therapy and prevention of cardiovascular diseases. ${ }^{19-21}$ Moreover, there is a growing interest in targeting hyaluronan-based delivery systems against eg atherosclerosis taking advantage of receptors for hyaluronic acid. ${ }^{22,23}$ However, despite many different approaches, the smart delivery of lipophilic vasoprotective molecules to the vascular wall remains a challenge including the difficulties to track their vascular delivery. ${ }^{5}$
DDSs may be tracked in vivo or ex vivo using fluorescence microscopy or ultrasonography. However, the application of fluorinated cargo molecules as probes have recently attracted significant attention for medical diagnosis and selective imaging as they can bring an unprecedented contrast in ${ }^{19} \mathrm{~F}$ Magnetic Resonance Imaging ( ${ }^{19} \mathrm{~F}$ MRI) or ${ }^{19} \mathrm{~F}$ Nuclear Magnetic Resonance ( ${ }^{19} \mathrm{~F}$ NMR) spectroscopy. A "second color" imaging has a wide range of advantages, ie ${ }^{19} \mathrm{~F}$ isotope has $100 \%$ natural abundance, a spin of $1 / 2$ and a gyromagnetic ratio comparable with a hydrogen atom (40.08 MHz/T for ${ }^{19} \mathrm{~F}$ and $42.58 \mathrm{MHz} / \mathrm{T}$ for $\left.{ }^{1} \mathrm{H}\right) .{ }^{24-26}$ Thus, ${ }^{19} \mathrm{~F}$ MRI has great potential as a method with a high contrast-to-noise ratio due to the lack of background signal and was applied in this work to detect very low concentrations of DDS ex vivo in the aorta. ${ }^{27}$

We proposed a novel application of recently developed and described nanocapsules, based on hyaluronic acid (HA), which is a ubiquitous glycosaminoglycan of the extracellular matrix and an integral part of the endothelial glycocalyx. Our previous works demonstrated that HyC12-based nanocapsules with liquid hydrophobic cores represent a promising and tunable nanodelivery system for lipophilic active compounds via both, oral and intravenous, administration in vivo. ${ }^{28,29}$ Interestingly, our in vitro studies revealed that nanocapsules were taken up by mouse liver sinusoidal endothelial cells and lung vascular endothelial cells, suggesting their possible application as a hydrophobic drug delivery system targeted to the vascular wall. ${ }^{28}$ Moreover, we showed also the potential for the delivery of lipophilic compounds due to their high stability and efficient uptake by various cells, especially the ones exposing CD44 receptors. The nanocapsules containing oleic acid cores were shown to act as an efficient anticancer agent while capsules with corn oil cores appeared to be nontoxic for various cell lines that imply their promising applicability. ${ }^{30}$ Therefore, the present work aimed to evaluate the possible use of hyaluronan-based nanocapsules for drug vascular delivery. For that purpose, we tested whether HyC12-based nanocapsules loaded with curcumin, administrated per os, would result in better hypotensive effects as compared with a low dose of curcumin given per os as a solution. Curcumin was chosen as a therapeutic agent, as its vasoprotective and hypotensive effects have been well documented in vivo, but only in relatively high doses, due to its poor water solubility and low bioavailability. ${ }^{17,31-34}$ Additionally, to confirm the delivery of studied nanocarriers to the vessel wall, novel fluorine-rich HyFC10-based nanocapsules were developed and administrated to rats per os, 
and their presence in the aorta was examined ex vivo using

${ }^{19} \mathrm{~F}$ NMR technique.

\section{Methods}

\section{Preparation of $\mathrm{HyCl}$ 2-Based}

Nanocapsules Loaded with Curcumin (HyCl2-Cur) and Fluorine-Rich

\section{HyFCI0-Based Nanocapsules}

\section{(HyFCl0-PFOB)}

\section{Modifications of Hyaluronic Acid}

Amphiphilic derivatives of hyaluronic acid (HA) were obtained by reacting HA sodium salt with either dodecylamine (C12) or perfluorodecylamine (FC10). The obtained HA derivatives - HyC12 and HyFC10, served as shells of nanocapsules. The synthesis of $\mathrm{HyC} 12$ was performed following the procedure described previously, ${ }^{28}$ while the modification of HA with perfluorinated alkyl chain (Figure 1) was done as described below.

$500 \mathrm{mg}$ of HA sodium salt $\left(\mathrm{M}_{\mathrm{w}} \approx 150000 \mathrm{~g} / \mathrm{mol}\right.$, SigmaAldrich) was dissolved in $100 \mathrm{~mL}$ of deionized water followed by the addition of $30 \mathrm{mg}$ of 1-ethyl-3-(3-dimethylaminopropyl)-carbodiimide (EDC, $>99 \%$, Sigma-Aldrich) and $18 \mathrm{mg}$ of N-hydroxysuccinimide (NHS, 98\%, SigmaAldrich). The mixture was diluted with $10 \mathrm{~mL}$ of water and vigorously stirred for 30 minutes. The reaction mixture was heated up to $37^{\circ} \mathrm{C}$ and $80 \mathrm{~mL}$ of tetrahydrofuran (THF, p.a., Avantor Performance Materials Poland), $20 \mathrm{~mL}$ of ethanol (96\%, p.a., Avantor Performance Materials Poland) and $1.55 \mathrm{~mL}$ of $0.1 \mathrm{M} \quad 1 \mathrm{H}, 1 \mathrm{H}, 2 \mathrm{H}, 2 \mathrm{H}$-perfluorodecylamine (FC10, Santa Cruz Biotechnology) in ethanol were added.
The reaction was carried out for $24 \mathrm{~h}$ under vigorous stirring at $37^{\circ} \mathrm{C}$ and followed by the dialysis of the reaction mixture against $1: 1 \mathrm{v} / \mathrm{v}$ solution of tert-butanol (99\%, Sigma-Aldrich) and PBS ( $\mathrm{pH}=7.4, \mathrm{c}=0.1 \mathrm{M}$, Sigma-Aldrich) for 3 days, and then against water for 4 days. The resulted derivative, HyFC10, was then freeze-dried.

To confirm modifications of HA and to calculate the degree of substitution (DS) by alkyl or perfluoroalkyl chains, the elemental analysis was applied. The DS values were determined based on carbon and nitrogen contents in the samples. A DS $=3.7 \%$ was obtained for $\mathrm{HyFC10}$ and comparable value of $\mathrm{DS}=3.0 \%$ was found for $\mathrm{HyC} 12$. Derivatization of HA using FC10 (HyFC10) was also confirmed by ${ }^{19} \mathrm{~F}$ NMR measurements (Avance III HD 400 $\mathrm{MHz}$ spectrometer, Bruker), by comparing the spectra of the FC10 substrate and the resulting HyFC10. All characteristic fluorine signals of $\mathrm{FC10}$ were found also in the spectrum of HyFC10 with only slight relative shifts (Figure S1).

\section{Formation of $\mathrm{HyCl}$ 2-Cur and $\mathrm{HyFClO}$-PFOB Nanocapsules}

The aqueous dispersion of the capsules with corn oil (Oil) core and $\mathrm{HyC} 12$ shells was obtained in a direct emulsification process, based on the mechanical shaking and sonication (Figure 2) as described previously with some minor changes. ${ }^{28}$ Briefly, an aqueous solution of HyC12 (1 g/L) was mixed with corn oil (Sigma-Aldrich) in 1000:3 ratio $(\mathrm{v} / \mathrm{v})$. Then, the mixture was shaken for 15 min using a vortex shaker and sonicated for $30 \mathrm{~min}$ at room temperature in the ultrasonic bath (40 W, Sonic-6, Polsonic). The nanocapsules loaded with curcumin (Cur, Sabinsa Europe
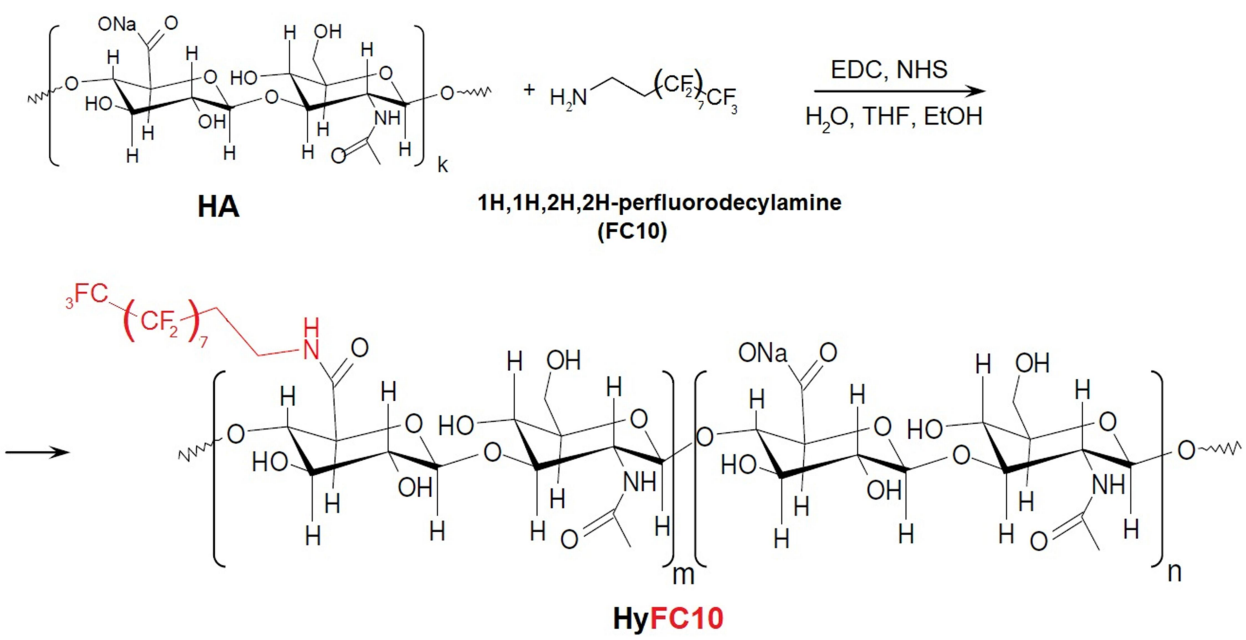

Figure I Scheme of hydrophobic modification of HA by perfluorinated alkyl chains. 


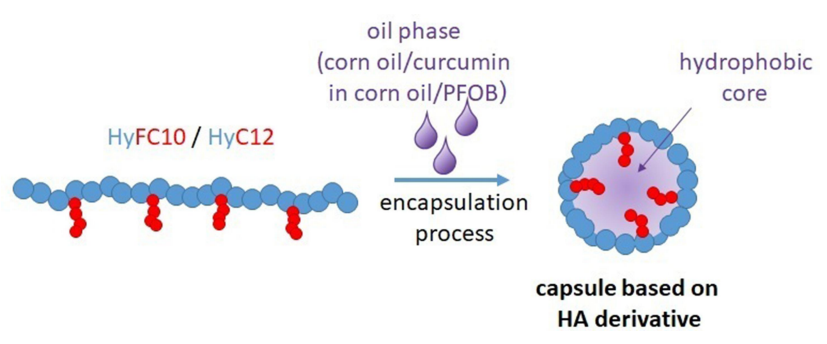

Figure 2 Scheme of the formation of the capsule.

$\mathrm{GmbH}$ ) were prepared in the same way using Cur solution in corn oil $(15 \mathrm{~g} / \mathrm{L})$ instead of pure oil.

The mechanical shaking and an ultrasound-assisted emulsification process with a few modifications were also applied to obtain capsules with liquid cores containing fluorine, HyFC10-PFOB (Figure 2). Namely, an aqueous solution of HyFC10 (2 g/L) was mixed with perfluorooctylbromide (PFOB, 99\%, Sigma-Aldrich), in a 1000:3 ratio $(\mathrm{v} / \mathrm{v})$. The mechanical shaking was prolonged up to $40 \mathrm{~min}$ following 20-minute sonication. Both steps were repeated.

The obtained HyFC10-PFOB capsules dispersed in water were characterized with ${ }^{19} \mathrm{~F}$ NMR to confirm the encapsulation and to estimate the encapsulation efficiency (EE) of PFOB. The sodium fluoride was added as a standard to the sample $(9 \mathrm{mM})$ and $\mathrm{EE}$ was calculated based on the integration of peaks of PFOB and ${ }^{19} \mathrm{~F}$ signal (Figure S2). The ${ }^{19} \mathrm{~F}$ NMR measurements revealed the $81 \%$ encapsulation efficiency of PFOB formulation, calculated based on the known amount of added $\mathrm{NaF}$ reference. The signals from HyFC10 shells of the capsules were not visible in the spectrum due to much lower content as compared to PFOB.

\section{Characterization of the Capsules}

Sizes and zeta potentials of the capsules dispersed in water were determined using Dynamic Light Scattering (DLS). The measurements were conducted at $22^{\circ} \mathrm{C}$ using a Malvern Zetasizer Nano ZS instrument working at $173^{\circ}$ detection angle. The general-purpose mode was used as the size distribution analysis algorithm, and the reported data represent the averages from three series of measurements (10-100 runs each) and their standard deviations (mean $\pm \mathrm{SD}, \mathrm{n}=3$ ).

The stability of the capsules in the digestive system was examined by their incubation in the simulated gastric juice $(0.2 \%(\mathrm{w} / \mathrm{v})$ sodium chloride (Sigma-Aldrich) in $0.7 \% \quad(\mathrm{v} / \mathrm{v})$ hydrochloric acid (35-38\%, Avantor Performance Materials Poland), $0.32 \%$ (w/v) pepsin
(Sigma-Aldrich), $\mathrm{pH}=1.8$ ) for $2 \mathrm{~h}$. Control samples were incubated with PBS. DLS measurements were performed in the given solutions to reveal the potential degradation of the capsules.

The influence of low $\mathrm{pH}$ conditions on the encapsulated Cur was studied as it might be not resistant to the acidity of the stomach (oral delivery). ${ }^{35,36}$ The HyC12-Cur capsules were incubated for $2 \mathrm{~h}$ in the solutions with $\mathrm{pH}$ adjusted to 7.4, 3.6, 1.8 and their sizes were measured using DLS. The fluorescence spectra of encapsulated and non-encapsulated Cur (dissolved in methanol (MeOH, p.a., Avantor Performance Materials Poland)) were also measured at neutral $(\mathrm{pH}=7.4)$ and acidic $(\mathrm{pH}=1.8)$ conditions.

The morphology of the formed capsules was observed by Scanning Electron Microscopy (SEM, Hitachi S-4700). For that purpose, model capsules with $\mathrm{HyC} 12$ and HyFC10 shells and n-octadecane (nC18, >99\%, GC, Sigma-Aldrich) cores were prepared. The capsules were obtained analogously to the previously described procedures, but the emulsification (10-minute mechanical shaking and 30 -minute sonication) was performed at $32^{\circ} \mathrm{C}$, above the melting point of $\mathrm{nC} 18$. After completing the procedure, the dispersions of the capsules were cooled to room temperature, which resulted in the solidification of nC18 cores of the capsules. Capsules with already solid cores were deposited on silicon substrates. The silicon wafer was prepared by cleaning with "piranha" solution (a mixture of $\mathrm{H}_{2} \mathrm{SO}_{4} \quad(96 \%$, Avantor Performance Materials Poland) and $\mathrm{H}_{2} \mathrm{O}_{2}(30 \%$, Avantor Performance Materials Poland), 1:1 (v/v)) followed by coating with cationic polyelectrolyte (immersion in polyethylenimine, PEI, branched, $\mathrm{M}_{\mathrm{w}} \approx 25,000 \mathrm{~g} / \mathrm{mol}$, Sigma-Aldrich, solution $(1 \mathrm{~g} / \mathrm{L})$ and sonication for $5 \mathrm{~min})$ to enhance the adhesion of negatively charged capsules. Then, the surface was carefully rinsed with water and dried in a stream of argon. Finally, the capsules with solid cores were deposited on the silicon wafer coated by PEI. The surface was again gently rinsed with water and dried in a stream of argon before SEM measurements.

\section{Animals}

The experiment involved male, hypertensive, heterozygous TGR(m-Ren2)27 rats ( $\mathrm{n}=6)$, obtained from Institute of Clinical and Experimental Medicine (Prague, Czech Republic), representing a unique transgenic model of the severe renin-angiotensin system (RAS)-dependent hypertension, in which the introduction of the murine renin transgene to the rat genome leads to the spontaneous 
increase in blood pressure. ${ }^{37}$ Normotensive Wistar rats $(\mathrm{n}=12)$ from Jagiellonian University Medical College, Faculty of Pharmacy (Krakow, Poland) were also used. After the delivery, animals undergo a few days of acclimatization followed by the handling procedure to tame rats with a touch. During the experiment, the hypertensive rats were kept in individual cages, according to the manufacturers' recommendations, to avoid the interference of telemetry signals and mutual damaging of the suture and transmitters after the implantation, leading to faster recovery. During the convalescence period, topical analgesic was applied to the wound to reduce animals' pain and distress after the surgery. Wistar rats were housed in colony cages, as they were used in a separate experiment. All animals were kept in individually-ventilated cages in controlled environmental conditions $\left(20-24^{\circ} \mathrm{C} ; 55 \% \pm 10 \%\right.$ of humidity; $12 \mathrm{~h}$ light/dark cycle) according to the European Union animal welfare regulations. Before and during entire experiments, rats had free access to food and water. Cages were additionally equipped with elements that enriched the environment: blocks for grinding teeth and rest houses. All procedures involving animals, including telemetry transmitters' implantation, compounds administration and sacrificing, were approved by the II Local Animal Ethics Commission at the Polish Academy of Sciences following the Ministry of Agriculture and Rural Development and European Union guidelines (permits no. 82/2018, 164/2018, 23/2019).

\section{Experimental Protocols}

Assessment of Hypotensive Effects of Curcumin-Rich $\mathrm{HyCl}$ 2-Based Nanocapsules as Compared with Curcumin Solution on the Progression of Hypertension in TGR(m-Ren2)27 Rats

The experiment was conducted in TGR(m-Ren2)27 rats $(n=6)$. At the age of 6 weeks, animals were separated into individual cages and implanted with telemetry blood pressure transmitters HD-S10 (Data Science International) to the abdominal aorta, according to the manufacturer's outlines with minor changes. Briefly, after an incision of abdominal skin and musculature, the abdominal aorta was exposed, and the catheter of the transmitter was introduced upstream into the vessel. The body of the transmitter was placed in the abdominal cavity and sutured. During the whole procedure, rats remainded under inhalation anesthesia with isoflurane (1-2\%). After the recovery period and baseline measurements, at the age of 8 weeks, when the hypertension was fully developed, the experiment was started and data (systolic blood pressure - SBP, diastolic blood pressure - DBP and heart rate - HR) was collected daily in 10 min intervals. Mean Arterial Pressure (MAP) was calculated based on SBP and DBP according to the formula: $\mathrm{MAP}=2 / 3 \mathrm{DBP}+1 / 3 \mathrm{SBP}$. Blood pressure and heart rate data were normalized to the first day values and expressed as an average change.

Rats were assigned into one group and received tested agents according to the following plan: 1st week - Control (no treatment), 2nd week - curcumin solution (Cur), 3rd week - washout (no treatment) and 4th week - HyC12Cur. Curcumin alone was prepared as a solution of curcumin in corn oil $(2 \mathrm{~g} / \mathrm{L})$ and administrated per gavage, while HyC12-Cur was given as a solution of encapsulated curcumin in drinking water $(45 \mathrm{mg} / \mathrm{L})$, which consumption was daily monitored. Different routes of administration were necessary to obtain an equal dose of curcumin $4.5 \mathrm{mg} / \mathrm{kg}$, in both experimental groups. The concentration of HyC12-Cur solution was adjusted to the daily water intake of TGR(m-Ren2)27 rats, which was precisely monitored for a couple of days before the experiment. TGR (m-Ren2)27 rats were characterized by the increased water intake (15 $\mathrm{mL} / 100 \mathrm{~g}$ b.w.), which is most likely a consequence of their hypertensive phenotype. The upregulated RAS system results in increased urination and water consumption, as Angiotensin II is physiologically involved in the regulation of body water homeostasis. ${ }^{38}$ Thus, to achieve the daily dose of $4.5 \mathrm{mg} / \mathrm{kg}$ b.w. of the curcumin the administration volume was adjusted to $10 \mathrm{~mL} / 100 \mathrm{~g}$ b.w., which guaranteed the full dose intake. In case the HyC12-Cur solution was consumed within $24 \mathrm{~h}$ the bottles were refilled with pure water to maintain free access to fluids.

\section{Detection of Fluorine-Rich HyFCl0-Based Nanocapsules in Aorta After Single Oral Administration}

Wistar rats (approx. $400 \mathrm{~g}$ b.w.) were assigned into 2 groups: control $(\mathrm{n}=8)$, receiving a vehicle (water) and HyFC10-PFOB group $(n=4)$, treated with fluorine-rich HyFC10-based nanocapsules dispersed in water to verify if nanocapsules based on HA derivative might be detected in a vascular wall. The aqueous dispersion of the capsules was administrated by gavage in a single dose, in a volume of $10 \mathrm{~mL} / \mathrm{kg} \mathrm{b.w.}$, and $8 \mathrm{~h}$ later animals were anesthetized by i.p. ketamine $(100 \mathrm{mg} / \mathrm{kg})$ - xylazine $(10 \mathrm{mg} / \mathrm{kg})$ injection and sacrificed. Afterward, the circulatory system was washed out with DPBS (Dulbecco, Gibco) and organs 
were fixed in situ using $4 \%$ buffered formalin perfusion to avoid vessels collapsing. Aorta was dissected and stored in formalin until further ex vivo detection of HyFC10-PFOB with ${ }^{19}$ F NMR spectroscopy analysis.

Ex vivo NMR measurements were performed using a horizontal 9.4T Bruker BioSpec 94/20 scanner. To provide a high SNR, a custom-built transmit-receive, double-resonant ${ }^{1} \mathrm{H}_{-}{ }^{19} \mathrm{~F}$, four-turn, $12 \mathrm{~mm}$ internal diameter strip-solenoid radio-frequency coil was used. For ${ }^{19} \mathrm{~F}$ NMR spectroscopy, excised intact aortas immersed in formalin were placed in $2 \mathrm{~mL}$ Eppendorf tubes together with a glass vial filled with $5 \mu \mathrm{L}$ $0.1 \%$ solution of 2,2,2-trifluoroethanol (TFE, Sigma Aldrich), containing approximately $200 \mathrm{nM}$ of fluorine atoms, as a fluorine concentration reference. The same reference vial was used for NMR measurements of all aorta samples. Additionally, to obtain the reference spectrum of HyFC10PFOB, the aqueous solution of the nanocapsules was analyzed. The following parameters of the Singlepulse sequence were used for measurements: the repetition time $3 \mathrm{~s}$, the number of averages 4800 and a total measurement time $4 \mathrm{~h}$.

\section{Statistical Analysis}

All results are presented as mean values \pm SD unless otherwise stated. The normality of data distribution and variances homogeneity were evaluated using ShapiroWilk and Levene's tests, respectively. Depending on the properties of variables parametric, Student's $t$-test or the non-parametric Mann-Whitney $U$-test was used to assess the statistical significance between the experimental and control groups at the level of $* \mathrm{P}<0.05, * * \mathrm{P}<0.01$, and $* * * \mathrm{P}<0.001$. Significant differences between studied groups are marked with $\dagger$. Calculations were performed using Statistica 12.0.

\section{Results}

\section{Physicochemical Characterization of} Nanocapsules Based on HA Derivatives

\section{Size and Zeta Potential}

The nanocapsules with liquid lipophilic cores, corn oil, and shells based on amphiphilic HA derivatives were fabricated (HyC12-Oil) and used as carriers of curcumin (HyC12-Cur). In addition, novel capsules with fluorinerich liquid cores made of $\mathrm{PFOB}$ and similar HA-based shells (HyFC10-PFOB) were formed for the first time and applied to confirm the vascular location of administrated capsules using ${ }^{19} \mathrm{~F}$ NMR. The physicochemical properties of formulations including size (hydrodynamic diameter) and zeta potentials were investigated using the DLS method.

All types of used oil-in-water formulations were characterized by the comparable hydrodynamic diameters of ca. $400 \mathrm{~nm}$ (Table 1). Similar distributions of the intensityweighted hydrodynamic diameters were found, but the narrowest one was observed for HyC12-Oil (Figure S3). Some differences were observed for zeta potential values that were found less negative for the capsules loaded with Cur and PFOB as compared to HyC12-Oil. Different cores used in the studied capsules (Oil, Cur and PFOB) influence the organization of the shell-forming macromolecules that affect the exposition of ionic groups at the capsules' surface. Moreover, the shell of HyFC10-PFOB capsules contained perfluorinated side chains that additionally influence the conformation of the shell macromolecules. However, in spite of different zeta potential values, all the capsules were found stable in an aqueous environment likely due to the contribution of other than electrostatic stabilizing factors (eg steric stabilization).

\section{Stability in Simulated Gastric Juice}

Low $\mathrm{pH}$ conditions present in the simulated gastric juice did not affect the stability of nanocapsules. Despite significantly different environments, samples did not exhibit macroscopic changes and had a very similar average size as well as low size distribution (PdI) (Figure S4). The slight broadening of the size distribution in the simulated gastric juice could be related to a different ionic strength and $\mathrm{pH}$ value of the environment. The lowest value of $\mathrm{pH}$ for these studies was selected based on the typical acidity of human stomach juice ( $\mathrm{pH}=1-2)$ as compared with $\mathrm{pH}$ of the rat stomach $(\mathrm{pH}=3-5) .{ }^{39}$

\section{Stability of Curcumin in Low pH Conditions}

Freshly prepared HyC12-Cur capsules in the environments with different $\mathrm{pH}$ values $(\mathrm{pH}=7.4,3.6$, and 1.8 , respectively for different $\mathrm{pH}$ values in human and rat stomach) exhibited practically the same average sizes and their distributions (Figure S5) ${ }^{39}$ After $2 \mathrm{~h}$ incubation, the average sizes slightly increased (the most in the lowest $\mathrm{pH}$ ) but no

Table I Average Hydrodynamic Diameters and Zeta Potentials Values of the Fabricated Oil-in-Water Formulations

\begin{tabular}{|l|c|c|c|}
\hline & HyCI 2-Oil & HyCI 2-Cur & HyFCI O-PFOB \\
\hline Diameter [nm] & $389 \pm 5$ & $403 \pm 20$ & $407 \pm 11$ \\
Zeta potential [mV] & $-29.1 \pm 0.3$ & $-17 \pm 1$ & $-17 \pm 1$ \\
\hline
\end{tabular}


additional fractions that could be assigned to the disintegration of the capsules were observed.

The fluorescence of encapsulated Cur stayed unaffected after changing the $\mathrm{pH}$ from 7.4 to 1.8 while free Cur dissolved in $\mathrm{MeOH}$ after adjusting $\mathrm{pH}$ to 1.8 by addition of $\mathrm{HCl}$ decreased significantly that may be related to its partial degradation (Figure S6).

\section{Morphology}

The nanocapsules were deposited on a silicon surface and visualized by SEM (Figure S7). The images of both systems, based on $\mathrm{HyC} 12$ and $\mathrm{HyFC10}$, revealed smooth spherical objects with diameters somewhat smaller than the ones determined via DLS. It can be rationalized as DLS measurements provide hydrodynamic diameters that are typically larger than the diameters of dry objects determined by SEM. Importantly, differences in viscosity, density, composition, phase of the cores that also imply variations of oil/water interfacial tension may result in different assembling of the polysaccharide forming the shells of the nanocapsules and also different sizes of those spherical objects. Nevertheless, the studied nanocapsules had comparable sizes regardless of the small differences between the applied HA derivatives.

\section{Hypotensive Effects of Low Dose Curcumin Administrated in $\mathrm{HyCl}$ 2-Based Capsules in TGR(m-Ren2)27 Hypertensive Rats}

Untreated, control animals were characterized by progressively increasing blood pressure for 7 days (Figure 3). Mean systolic (SBP) and diastolic blood pressure (DBP) rose gradually $(\sim 7 \pm 4 \mathrm{mmHg} / \mathrm{day})$ and reached $192 \pm 14$ $\mathrm{mmHg}$ and $136 \pm 15 \mathrm{mmHg}$, respectively. The application of curcumin solution in a low dose of $4.5 \mathrm{mg} / \mathrm{kg}$ did not affect the progression of hypertension, as SBP, DBP and MAP were not reduced significantly in comparison with the control after 7 days of the treatment (Figure 3). In contrast, the administration of HyC12-Cur, in the same low dose of $4.5 \mathrm{mg} / \mathrm{kg}$, limited the increase in SBP, DBP and MAP. The earliest changes occurred on the 2nd day of the treatment in DBP, while the reduction of SBP, and MAP was observed starting from the 4th day of the treatment. After 7 days of the treatment, the changes in mean SBP, DBP, and MAP were $-2.0 \pm 0.8 \mathrm{mmHg},-3.9 \pm 0.7$ $\mathrm{mmHg}$ and $-3.3 \pm 0.7 \mathrm{mmHg}$, respectively, as compared with the control (Figure 4). HyC12-Cur had no effects on heart rate.

\section{${ }^{19} \mathrm{~F}$ NMR-Based Characteristics and Detection of HyFCI0-PFOB in the Vascular Wall of the Rat Aorta After Single Oral Administration}

HyFC10-PFOB capsules measured using ${ }^{19} \mathrm{~F}$ NMR displayed characteristic peaks of $\mathrm{PFOB}$ : $-\mathrm{CF}_{2} \mathrm{Br}$ located at $-66 \mathrm{ppm},-\mathrm{CF}_{3}$ located at $-84 \mathrm{ppm}$, while multiplet around $124 \mathrm{ppm}$ was assigned to $-\mathrm{CF}_{2^{-}}$groups (see Figure 5 and Figure S2 for detailed assignments). The peak at -78.8 ppm was assigned to $-\mathrm{CF}_{3}$ group of TFE ( $\left.\delta=77.86 \mathrm{ppm}\right)$, which was chosen as a convenient reference as its signal is well-separated from all PFOB peaks.

To confirm the delivery of HyFC10-PFOB to the vascular wall, ${ }^{19} \mathrm{~F}$ NMR spectra of the aortas taken from the rats subjected to a single injection of HyFC10-PFOB nanocapsules (see methods for details) were measured and compared with ${ }^{19} \mathrm{~F}$ NMR signal from the aorta taken from the control rats. Two measurements were rejected in the control group due to artifacts, leaving $n=6$. In the case of HyFC10-PFOB treated animals, one measurement was rejected (no fluorine was detected), leaving $n=3$. In aortas from the control group, no fluorine was detected, while in the spectra of HyFC10-PFOB treated rats, two main peaks located at $-81.6 \mathrm{ppm}$ and $-87.6 \mathrm{ppm}$, symmetrically around the position of $\mathrm{CF}_{3}$ peak in the spectra of HyFC10PFOB capsules were found (Figure 5). The small residual peaks were also observed at $\sim-65 \mathrm{ppm}$ and around -124 ppm, close to the positions of $\mathrm{CF}_{2} \mathrm{Br}$ and multiplet of $\mathrm{CF}_{2}$ in the original spectrum $\mathrm{HyFC} 10-\mathrm{PFOB}$, respectively. However, as these peaks were close to the limit of detection, we considered them negligible.

\section{Discussion}

In the present work, we demonstrated that in hypertensive TGR(m-Ren2)27 rats, a representative model of RASdependent hypertension, ${ }^{40-42}$ a low dose of curcumin (4.5 mg/kg) administrated in HyC12-based nanocapsules, resulted in mild hypotensive effects, visible as a gradual fall in SBP, DBP and MAP. In contrast, a low dose of curcumin given as the oil solution at the same dose of $4.5 \mathrm{mg} / \mathrm{kg}$ was ineffective. Although we do not have direct evidence of the curcumin delivery to the vascular wall, in a proof-of-concept study, taking advantage of MRI-based detection of purposely synthesized HyFC10-PFOB, we 
$\rightarrow$ Control $\rightarrow$ Cur $\rightarrow$ HyC12-Cur

$\Delta$ SBP

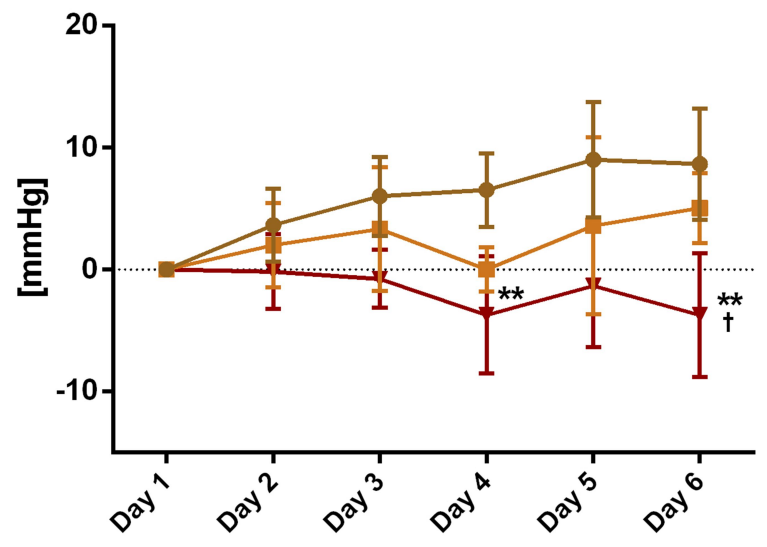

$\triangle$ MAP

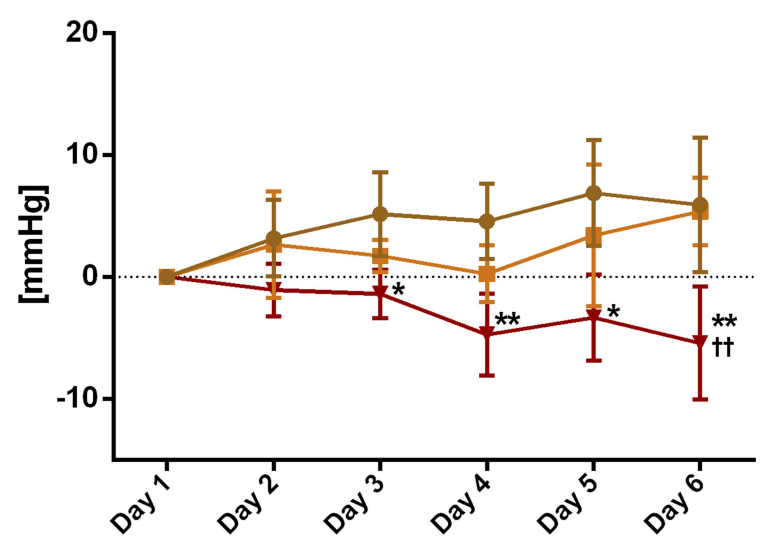

$\Delta \mathrm{DBP}$

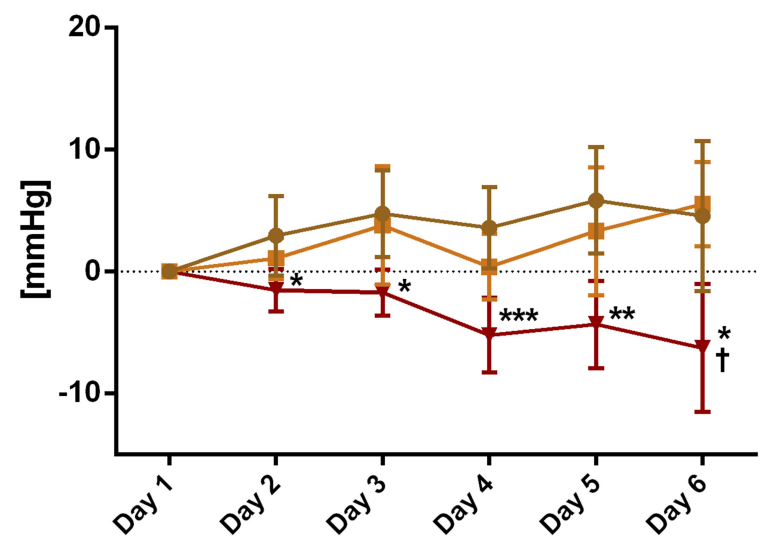

$\Delta \mathrm{HR}$

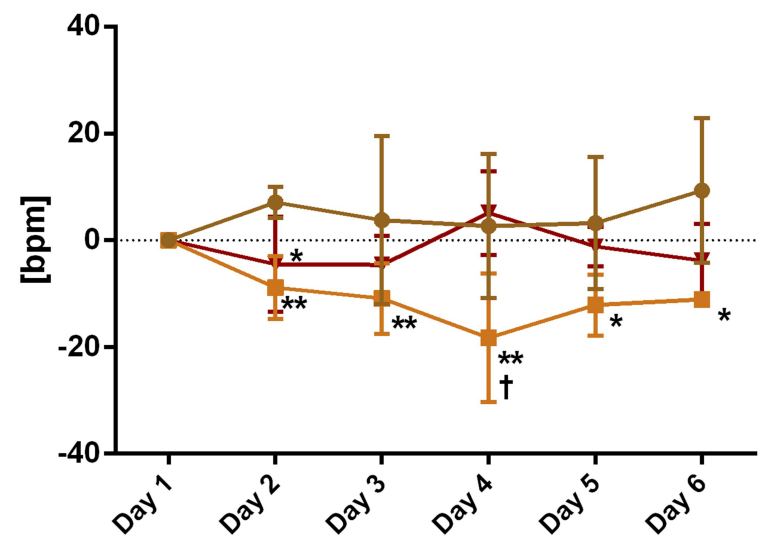

Figure 3 Progressive changes in SBP, DBP, MAP and HR in hypertensive TGR(m-Ren2)27 rats after administration of HyCl2-Cur, curcumin in solution or in controls. Notes: Statistical significance between the experimental and control groups is marked with asterisks: $* \mathrm{P}<0.05$, $* * \mathrm{P}<0.0 \mathrm{I}$, and $* * * \mathrm{P}<0.00 \mathrm{I}$. Significant differences between studied groups are marked with ${ }^{\dagger}\left({ }^{\dagger} \mathrm{P}<0.05,{ }^{\dagger \dagger} \mathrm{P}<0.0 \mathrm{I}\right)$.

Abbreviations: $\triangle \mathrm{SBP}$, change in systolic blood pressure; $\triangle \mathrm{DBP}$, change in diastolic blood pressure; $\triangle \mathrm{MAP}$, change in mean arterial pressure; $\triangle \mathrm{HR}$, change in heart rate.

demonstrated that the components of nanocapsules were detected in aorta after a single administration of HyFC10PFOB by oral gavage. Altogether, this study provides preliminary evidence that the nanocapsules composed of hyaluronan, a ubiquitous glycosaminoglycan of the extracellular matrix, and an integral part of endothelial glycocalyx, may represent a suitable approach to deliver hydrophobic, poorly bioavailable compounds, to the vascular wall. This conclusion stays in line with our previous studies showing the uptake of nanocapsules based on HA derivatives by endothelial cells in vitro, ${ }^{28}$ supporting their possible application for targeted vascular delivery.

Curcumin is a naturally occurring polyphenolic compound, widely consumed with a diet that exerts beneficial vasoprotective effects. Several studies demonstrated that prolonged administration of curcumin attenuated hypertension development, improved arterial elasticity, reduced oxidative stress, and preserved endothelial function in both, animals and humans. $^{31-33,43-46}$ In various models of hypertension, curcumin elicited hypotensive actions attributed to numerous molecular targets, including restoring eNOS function, increasing glutathione plasma levels or down-regulation of angiotensinconverting enzyme (ACE) and angiotensin II (Ang II) type-1 receptor. ${ }^{31,33,46}$ Unfortunately, due to the poor water solubility and low bioavailability of curcumin, as a consequence of its low intestine absorption and rapid metabolism, ${ }^{47}$ vasoprotective effects of curcumin could only be achieved with very high doses (50-1000 mg/kg) and special formulations. 


\section{$\Delta$ SBP per group}

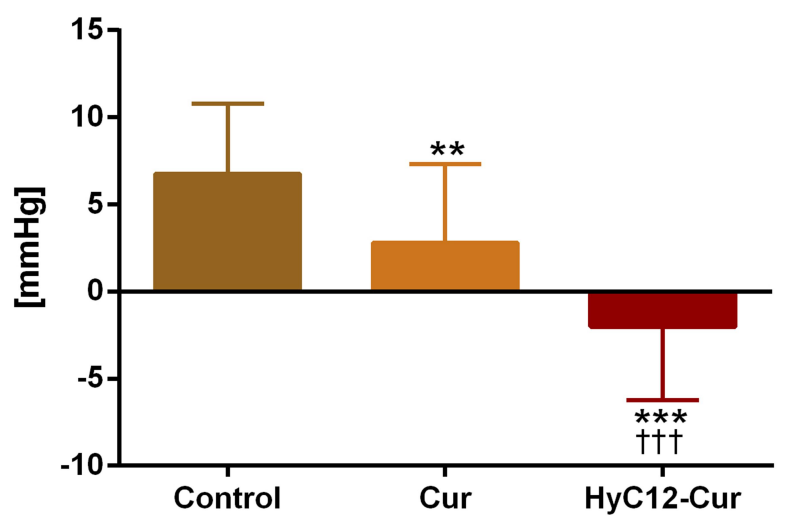

$\Delta$ MAP per group

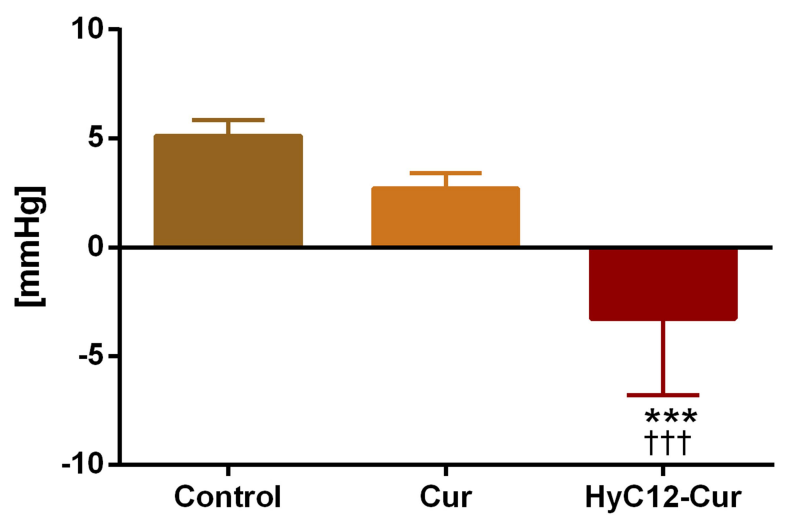

$\Delta$ DBP per group

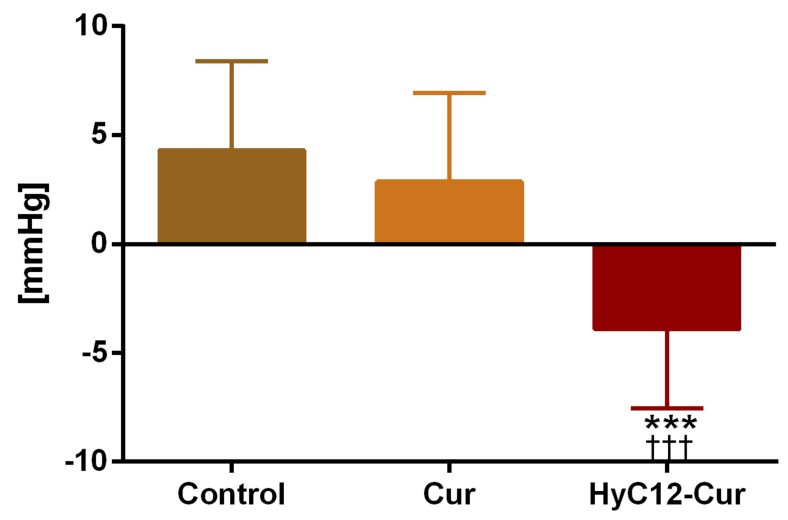

$\Delta$ HR per group

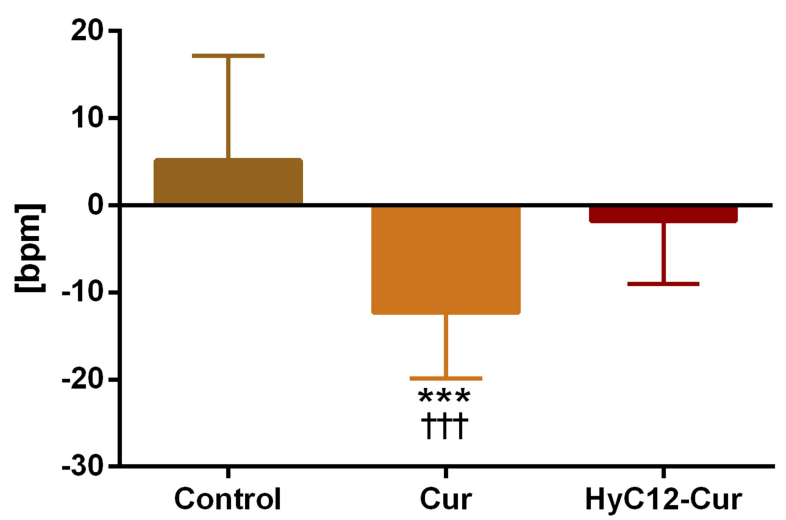

Figure 4 Changes in SBP, DBP, MAP and HR in hypertensive TGR(m-Ren2)27 rats after 7 days of HyCl2-Cur administration as compared to curcumin administered in solution and to controls.

Notes: Statistical significance between the experimental and control groups is marked with asterisks: **P $<0.01$ and $* * * \mathrm{P}<0.00 \mathrm{I}$. Significant differences between studied groups are marked with ${ }^{\dagger}\left({ }^{\dagger \dagger} \mathrm{P}<0.00 \mathrm{I}\right)$.

Abbreviations: $\triangle \mathrm{SBP}$, change in systolic blood pressure; $\triangle \mathrm{DBP}$, change in diastolic blood pressure; $\triangle \mathrm{MAP}$, change in mean arterial pressure; $\triangle \mathrm{HR}$, change in heart rate.

Our experiment was performed in TGR(m-Ren2)27 rats, representing a unique model of RAS-dependent hypertension, relevant to the human pathology. ${ }^{40-42}$ Furthermore, therapy of TGR(m-Ren2)27 rats with drugs commonly used to treat hypertension in humans, such as calcium channel blockers or ACE inhibitors, suppressed blood pressure elevation in this model, ${ }^{37,48,49}$ supporting its relevance to the pathophysiology of human hypertension. Accordingly, the mild hypotensive effect of curcumin enclosed in HyC12-based nanocapsules shown in this work in TGR(m-Ren2)27 rats suggests that HyC12-Cur may also have a therapeutic significance for humans.

The most important finding of this study was the hypotensive effect of encapsulated curcumin, given in a low dose of $4.5 \mathrm{mg} / \mathrm{kg}$ that was at least partially ascribed to its direct delivery to the vessel wall. Although we did not provide definitive evidence for the targeted vascular delivery of curcumin in HyC12-Cur, the ${ }^{19} \mathrm{~F}$ NMR - based detection of capsules' components in rat aortas seems to confirm that nanocarriers based on HA derivatives were targeted to the vascular wall. Indeed, in the current study, taking advantage of the specially designed fluorine-rich HyFC10-PFOB nanocapsules we established a novel methodology for the ex vivo detection of HA-based nanocapsules using ${ }^{19} \mathrm{~F}$ NMR. For that purpose, HA was modified by attaching perfluorinated alkyl chain leading to the formation of HAderivative with similar content of hydrophobic chains as in the case of HyC12. Liquid cores of HyFC10-based nanocapsules were made of PFOB (the perfluorinated oil), which is intensively studied in ultrasound or ${ }^{19} \mathrm{~F}$ MRI imaging, as well as an artificial oxygen carrier, due to its biocompatibility and good tolerance in both animals and humans. ${ }^{50}$ 


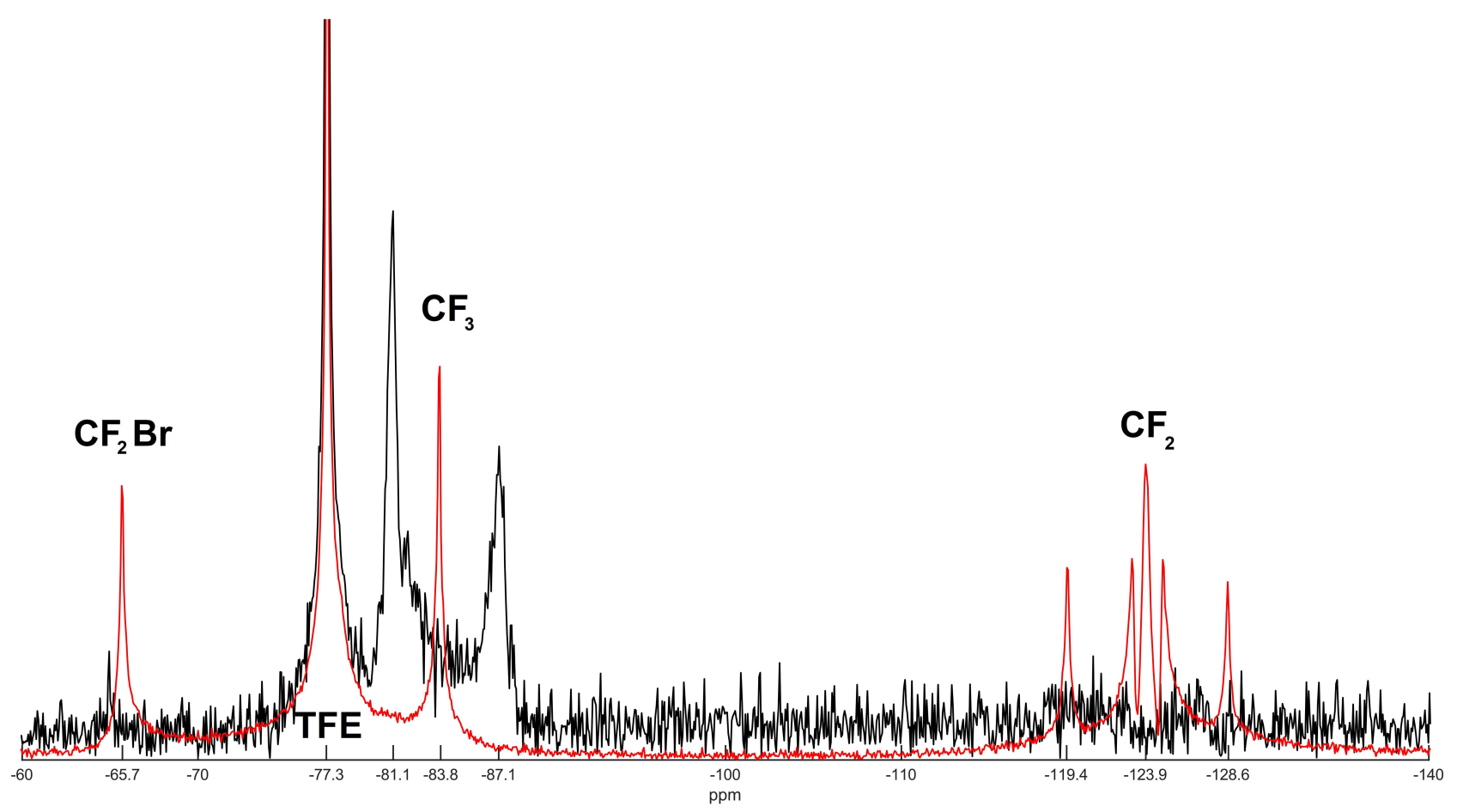

Figure 5 Representative ${ }^{19} \mathrm{~F}$ NMR spectrum of an excised rat aorta (black) overlaid on a HyFCl0-PFOB solution spectrum (red). Note: Both spectra were measured with TFE as a reference $\left(\delta=-77.3 \mathrm{ppm}\right.$ relative to $\left.\mathrm{CFCl}_{3}\right)$ using the Bruker BioSpec $94 / 20$ scanner.

According to ${ }^{19} \mathrm{~F}$ NMR analysis, the encapsulation efficacy of PFOB in HyFC10-based capsules was as high as $81 \%$. Given the physicochemical properties of PFOB, ie its partition coefficient, $\log \mathrm{P}=6.9$ and extremely low water solubility $\left(10^{-9} \mathrm{M}\right)$, resulting in very poor bioavailability, it is clear that administration of $\mathrm{PFOB}$ requires encapsulation or preparation of an aqueous emulsion. Thus, all the observed fluorine content in a given tissue upon administration of HyFC10-PFOB capsules should come from the encapsulated PFOB. Components of HyFC10-PFOB capsules were detected in the vascular wall of aortas using ${ }^{19} \mathrm{~F}$ NMR spectroscopy. The exact origin of the two main ${ }^{19} \mathrm{~F}$ peaks $(-81.1$ and $-87.1 \mathrm{ppm})$ observed in the aorta samples (Figure 5) is not clear, due to the significant alteration of the fluorine spectra, but their positions suggest the terminal $\mathrm{CF}_{3}$ groups in $\mathrm{PFOB}$ as an origin $(-83.8 \mathrm{ppm})$. The split of this peak into two, as well as significant broadening of the spectra lines, may result from different local environments in the vicinity of the terminal $\mathrm{CF}_{3}$ groups, or local magnetic field inhomogeneity due to aorta microstructure. The shift of the spectrum peaks in model biological microenvironments was reported previously as a result of the interaction of PFOB with POPC bilayer, as well as hexane molecules. ${ }^{51}$ Strong reduction of the signals originating from $\mathrm{CF}_{2} \mathrm{Br}$ and $\mathrm{CF}_{2}$ groups may be attributed to the immobilization of the
PFOB backbone, which results in broadening of the spectrum and limited detection at the experimental conditions. Thus, the observed spectral features may be related to the disintegration of nanocapsules after their delivery and incorporation of PFOB into the aorta tissue, combined with immobilization of their molecular backbone. The terminal $\mathrm{CF}_{3}$ groups may remain relatively more mobile distributed in two chemically/physically different surroundings that resulted also in two chemical shifts of the signal. Based on that assumption, integration of the peaks' areas as compared to the integrated signal from the reference peak, allowed the calculation of the average PFOB concentration in the tissue reaching $0.36 \pm 0.06 \mu \mathrm{M}$ PFOB per $1 \mathrm{~g}$ of the aorta (based on wet mass calculation).

Herein, we demonstrated that hyaluronic acid-based capsules, depending on the applied liquid core and with minor shell modifications, could serve either for the delivery of active compounds or as a carrier of the perfluorinated contrast agent. The outer shell chemistry and fundamental physicochemical characteristics were comparable between the obtained capsules (HyC12-Oil, HyC12-Cur and HyC12-PFOB) that imply similar interactions with the biological environment, despite differences in the core composition. Our previous in vitro studies demonstrated efficient cellular uptake of HyC12-based nanocapsules 
and intracellular release of the cargo thanks to the presence of hyaluronidase, an enzyme cutting HA-based chains. ${ }^{30}$ Thus, together with the current results confirming their stability in simulated gastric juice, it indicates that nanocapsules are stable in extracellular fluid and the inner environment of the capsules can affect the cells only after enzymatic degradation of the HA-based shells inside the cells. In fact, the capsules seem to protect curcumin dissolved in their cores against the acidic environment present in the stomach. Interestingly, the previous pharmacokinetic study in vivo using fluorescent probes revealed that HyC12-based nanocapsules, administrated to mice in a single dose, were accumulated preferentially in livers and lungs, with even greater intensity in the endotoxemia. ${ }^{28}$ Given the fact that endotoxemia is associated with the glycocalyx shedding and the overexpression of endothelial hyaluronic receptors, these results may suggest the interaction between $\mathrm{HyC} 12$-based nanocapsules and the endothelium. However, possible mechanisms have not been investigated yet. The shell of $\mathrm{HyC} 12$ nanocapsules is based on HA, the natural polysaccharide and the glycocalyx constituent, recognized by hyaluronan receptors including CD44 and Hyaluronic Acid Receptor for Endocytosis (HARE) and uptaken from the bloodstream. ${ }^{52}$ CD44 and HARE are mainly located on the surface of endothelial cells and macrophages in the vascular wall and their overexpression is observed during inflammatory processes associated with many diseases including sepsis, ischemia-reperfusion, and atherosclerosis, becoming a promising target for vascular drug delivery. ${ }^{22,53}$ Recent studies in animal models of atherosclerosis have shown that HA-based nanoparticles were selectively uptaken through the CD44 and HARE receptors overexpressed in atherosclerotic plaque in the vascular wall, resulting in therapeutic effects. ${ }^{22,23,54}$ Furthermore, Hou et al demonstrated that the treatment of high fat diet-fed rats with curcumin enclosed in CD44-specific HA-based micelles for 2 weeks reduced the area of atherosclerotic lesions in rats' aortas supporting a possibility of the vascular delivery of HA-based nanocarriers. ${ }^{55}$ Accordingly, hypotensive effects of HyC12-Cur observed in this study, as well as the detection of HyFC10-PFOB components in rat aortas, might indeed be due to the HA-based uptake of HyC12based nanocapsules to the vascular wall.

Moreover, HyC12-Cur did not affect the heart function, while administration of curcumin solution resulted in a decrease of HR without influencing the blood pressure. These different effects might be explained by the distinct biodistribution of Cur solution as compared with HyC12Cur, which additionally supports the targeted delivery of nanocapsules to the vessel wall. In fact, curcumin was cardioprotective by improving heart function, without affecting the blood pressure, in rats with myocardial infarction. ${ }^{56}$ Moreover, the administration of the curcumin directly to the isolated perfused rat hearts evoked negative chronotropic and inotropic effects. ${ }^{57}$ Although the exact mechanism behind these actions has not yet been elucidated, it was proven by the pharmacokinetic study that curcumin accumulates in rat hearts, even after single-dose administration. ${ }^{58}$ These results stay in line with our observations and suggest that curcumin given in solution might be uptaken by the cardiomyocytes and thus directly affect the heart rate. In turn, the shell composition of $\mathrm{HyC} 12-\mathrm{Cur}$ might enhance nanocapsules affinity to the endothelial cells, resulting in $\mathrm{HyCl2}$-Cur accumulation in the vascular wall. Thus, it might well be that HyC12-Cur as compared with Cur solution had distinct effects on vascular and heart function.

On the other hand, the efficacy of encapsulated curcumin might be also related to improved bioavailability and increased absorption from the gastrointestinal tract. Indeed, our recent in vivo study brought the evidence that $\mathrm{HyC} 12$-based nanocapsules were well absorbed from the gastrointestinal tract and distributed through the circulation after oral administration. ${ }^{28}$ Thus, enclosing curcumin in HyC12-Cur may improve its bioavailability and prolong the clearance time, enhancing its therapeutic potential even at low doses.

\section{Conclusions}

To sum up, the nanocapsules composed of hyaluronatebased shell and liquid oleic core represent a suitable approach to deliver hydrophobic, poorly bioavailable compounds to the vascular wall as exemplified here by the demonstration of the hypotensive effect in TGR (m-Ren2)27 rats of low dose curcumin encapsulated in such nanocapsules (HyC12-Cur). Nanocapsules with perfluorinated cores (HyFC10-PFOB) having comparable sizes and the shell composition to original HyC12-based capsules were specially designed to confirm by ${ }^{19} \mathrm{~F}$ NMR spectroscopy the presence of the cores of the HyFC10PFOB capsules in the vascular walls, after a single administration. Altogether, this work demonstrated vascular delivery of HyC12-based capsules, though the increased pharmacological efficacy of encapsulated curcumin might be also related to the improved bioavailability of curcumin 
administrated in HyC12-Cur. Obviously, further optimization and mechanistically oriented studies are needed to better understand the mechanisms of the uptake and retention of nanocarriers based on HA derivatives in the vascular wall.

\section{Supplementary Materials}

Supplementary information accompanies this paper.

\section{Ethics Approval and Consent to Participate}

All procedures involving animals were approved by the II Local Animal Ethics Commission at the Polish Academy of Sciences (permits no. 82/2018, 164/2018, 23/2019).

\section{Funding}

This work was supported by the National Science Centre (Grant No. DEC-2015/16/W/NZ4/00070) and the Ministry of Science and Higher Education of the Republic of Poland (Grant No. DI2015 015445). The open-access publication of this article was funded by the Priority Research Area BioS under the program "Excellence Initiative Research University" at the Jagiellonian University in Krakow.

\section{Disclosure}

The authors declare no conflicts of interest in this work.

\section{References}

1. Muzykantov V, Muro S. Targeting delivery of drugs in the vascular system. Int J Transp Phenom. 2011;12:41-49.

2. Loftsson T, Brewster ME. Pharmaceutical applications of cyclodextrins: basic science and product development. J Pharm Pharmacol. 2010;62:1607-1621. doi:10.1111/j.2042-7158.2010.01030.x

3. Muro S, Muzykantov V. Targeting of antioxidant and anti-thrombotic drugs to endothelial cell adhesion molecules. Curr Pharm Des. 2005;11:2383-2401. doi:10.2174/1381612054367274

4. Savjani KT, Gajjar AK, Savjani JK. Drug solubility: importance and enhancement techniques. ISRN Pharm. 2012;2012:1-10.

5. Ma Z, Wang N, He H, Tang X. Pharmaceutical strategies of improving oral systemic bioavailability of curcumin for clinical application. $J$ Control Release. 2019;316:359-380. doi:10.1016/j.jconrel.201 .9.10.053

6. Lombardo D, Kiselev MA, Caccamo MT. Smart nanoparticles for drug delivery application: development of versatile nanocarrier platforms in biotechnology and nanomedicine. J Nanomater. 2019;2019:3702518. doi: $10.1155 / 2019 / 3702518$

7. Kiseleva RY, Glassman PM, Greineder CF, Hood ED, Shuvaev VV, Muzykantov VR. Targeting therapeutics to endothelium: are we there yet? Drug Deliv Transl Res. 2018;8:883-902. doi:10.1007/s13346017-0464-6

8. Singh AP, Biswas A. Targeted therapy in chronic diseases using nanomaterial-based drug delivery vehicles. Signal Transduct Target Ther. 2019;4:33.
9. Johnsen KB, Bak M, Kempen PJ, et al. Antibody affinity and valency impact brain uptake of transferrin receptor-targeted gold nanoparticles. Theranostics. 2018;8:3416-3436. doi:10.7150/thno.25228

10. Peng S, Wang Y, Li N, Li C. Enhanced cellular uptake and tumor penetration of nanoparticles by imprinting the "hidden" part of membrane receptors for targeted drug delivery. Chem Commun. 2017;53:11114-11117. doi:10.1039/C7CC05894B

11. Koren E, Torchilin VP. Drug carriers for vascular drug delivery. IUBMB Life. 2011;63:586-595. doi:10.1002/iub.496

12. Shuvaev VV, Han J, Yu KJ, et al. PECAM-targeted delivery of SOD inhibits endothelial inflammatory response. FASEB J. 2011;25:348-357. doi:10.1096/fj.10-169789

13. Muro S, Garnacho C, Champion JA, et al. Control of endothelial targeting and intracellular delivery of therapeutic enzymes by modulating the size and shape of ICAM-1-targeted carriers. Mol Ther. 2008;16:1450-1458. doi:10.1038/mt.2008.127

14. Simone E, Sen Ding B, Muzykantov V. Targeted delivery of therapeutics to endothelium. Cell Tissue Res. 2009;335:283-300. doi:10.1007/s00441-008-0676-7

15. Torchilin VP, Levchenko TS, Lukyanov AN, et al. p-nitrophenylcarbonyl-PEG-PE-liposomes: fast and simple attachment of specific ligands, including monoclonal antibodies, to distal ends of PEG chains via p-nitrophenylcarbonyl groups. Biochim Biophys Acta. 2001;1511:397-411. doi:10.1016/S0005-2728(01)00165-7

16. Pasut G, Veronese FM. State of the art in PEGylation: the great versatility achieved after forty years of research. $J$ Control Release. 2012;161:461-472. doi:10.1016/j.jconrel.2011.10.037

17. Bansal S, Goel M, Aqil F, Vadhanam MV, Gupta RC. Advanced drug-delivery systems of curcumin for cancer chemoprevention. Cancer Prev Res. 2011;4:1158-1171. doi:10.1158/1940-6207.CAPR-10-0006

18. Sakurai Y, Akita H, Harashima H. Targeting tumor endothelial cells with nanoparticles. Int J Mol Sci. 2019;20:1-15. doi:10.3390/ijms20235819

19. Aggarwal BB, Shishodia S. Molecular targets of dietary agents for prevention and therapy of cancer. Biochem Pharmacol. 2006;71:1397-1421.

20. Goel A, Kunnumakkara AB, Aggarwal BB. Curcumin as "Curecumin": from kitchen to clinic. Biochem Pharmacol. 2008;75:787-809. doi:10.1016/j.bcp.2007.08.016

21. Amri A, Chaumeil JC, Sfar S, Charrueau C. Administration of resveratrol: what formulation solutions to bioavailability limitations? J Control Release. 2012;158:182-193. doi:10.1016/j. jconrel.2011.09.083

22. Lee GY, Kim JH, Choi KY, et al. Hyaluronic acid nanoparticles for active targeting atherosclerosis. Biomaterials. 2015;53:341-348. doi:10.1016/j.biomaterials.2015.02.089

23. Zhang $\mathrm{M}$, He J, Jiang $\mathrm{C}$, et al. Plaque-hyaluronidase-responsive high-density- lipoprotein-mimetic nanoparticles for multistage intimal-macrophage-targeted drug delivery and enhanced anti-atherosclerotic therapy. Int $J$ Nanomedicine. 2017;12:533-558. doi:10.2147/IJN.S124252

24. Tirotta I, Dichiarante V, Pigliacelli C, et al. 19F magnetic resonance imaging (MRI): from design of materials to clinical applications. Chem Rev. 2015;115:1106-1129. doi:10.1021/cr500286d

25. Bachert P. Pharmacokinetics using fluorine NMR in vivo. Prog Nucl Magn Reson Spectrosc. 1998;33:1-56. doi:10.1016/S0079-6565(98) 00016-8

26. Reid DG, Murphy PS. Fluorine magnetic resonance in vivo: a powerful tool in the study of drug distribution and metabolism. Drug Discov Today. 2008;13:473-480. doi:10.1016/j. drudis.2007.12.011

27. Ruiz-Cabello J, Barnett BP, Bottomley PA, Bulte JWM. Fluorine (19F) MRS and MRI in biomedicine. NMR Biomed. 2011;24:114-129. doi:10.1002/nbm.1570

28. Szafraniec J, Błażejczyk A, Kus E, et al. Robust oil-core nanocapsules with hyaluronate-based shells as promising nanovehicles for lipophilic compounds. Nanoscale. 2017;9:18867-18880. doi:10.1039/C7NR05851A 
29. Szafraniec-Szczęsny J, Janik-Hazuka M, Odrobińska J, Zapotoczny S Polymer capsules with hydrophobic liquid cores as functional nanocarriers. Polymers. 2020;12:1999. doi:10.3390/polym12091999

30. Janik-Hazuka M, Szafraniec-Szczęsny J, Kamiński K, Odrobińska J, Zapotoczny S. Uptake and in vitro anticancer activity of oleic acid delivered in nanocapsules stabilized by amphiphilic derivatives of hyaluronic acid and chitosan. Int $J$ Biol Macromol. 2020;164:2000-2009. doi:10.1016/j.ijbiomac.2020.07.288

31. Boonla O, Kukongviriyapan U, Pakdeechote P. Nitric oxide curcumin improves endothelial dysfunction and vascular remodeling in $2 \mathrm{~K}-1 \mathrm{C}$ hypertensive rats by raising nitric oxide availability and reducing oxidative stress. Nitric Oxide. 2014;42:44-53. doi:10.1016/j.niox.2014.09.001

32. Hlavačková L, Janegová A, Uličná O, Janega P, Černá A, Babál P. Spice up the hypertension diet - Curcumin and piperine prevent remodeling of aorta in experimental L-NAME induced hypertension. Nutr Metab. 2011;8:1-10. doi:10.1186/1743-7075-8-72

33. Nakmareong S, Kukongviriyapan U, Pakdeechote $P$, et al. Antioxidant and vascular protective effects of curcumin and tetrahydrocurcumin in rats with L-NAME-induced hypertension. Naunyn Schmiedebergs Arch Pharmacol. 2011;383:519-529. doi:10.1007/ s00210-011-0624-z

34. Anand P, Kunnumakkara AB, Newman RA, Aggarwal BB. Bioavailability of curcumin: problems and promises. Mol Pharm. 2007;4:807-818. doi:10.1021/mp700113r

35. Khart M, Du Z, Zhang G, McClements DJ. Physical and chemical stability of curcumin in aqueous solutions and emulsions: impact of pH, temperature, and molecular environment. J Agric Food Chem. 2017;65:1525-1532. doi:10.1021/acs.jafc.6b04815

36. Wang YJ, Pan MH, Cheng AL, et al. Stability of curcumin in buffer solutions and characterization of its degradation products. J Parmaceut Biomed. 1997;15:1867-1876. doi:10.1016/S0731-7085(96)02024-9

37. Witte K, Huser L, Knotter B, Heckmann M, Schiffer S, Lemmer B. Normalisation of blood pressure in hypertensive TGR(mREN2)27 rats by amlodipine vs. enalapril: effects on cardiac hypertrophy and signal transduction pathways. Naunyn Schmiedebergs Arch Pharmacol. 2001;363:101-109. doi:10.1007/s002100000343

38. Krsková L, Vrabcová M, Talarovicová A, Zeman M. Influence of up-regulated renin-angiotensin system on the exploration, anxiety-related behavior and object recognition. Acta Biol Hung. 2009;60(4):369-383. doi:10.1556/ABiol.60.2009.4.4

39. Hatton GB, Yadav V, Basit AW, Merchant HA. Animal Farm: considerations in animal gastrointestinal physiology and relevance to drug delivery in humans. J Pharm Sci. 2015;104:2747-2776. doi:10.1002/jps.24365

40. Řezáčová L, Hojná S, Kopkan L, et al. Role of angiotensin II in chronic blood pressure control of heterozygous Ren- 2 transgenic rats: peripheral vasoconstriction versus central sympathoexcitation. Biomed Pharmacother. 2019;116:108996. doi:10.1016/j.biopha.2019.108996

41. Vacková Š, Kikerlová S, Melenovsky V, et al. Altered renal vascular responsiveness to vasoactive agents in rats with angiotensin II-dependent hypertension and congestive heart failure. Kidney Blood Press Res. 2019;44:792-809. doi:10.1159/000501688

42. Feng Y, Wang Y, Stock O, et al. Vasoregression linked to neuronal damage in the rat with defect of polycystin-2. PLoS One. 2009;4: e7328. doi:10.1371/journal.pone.0007328

43. Campbell MS, Berrones AJ, Krishnakumar IM, Charnigo RJ, Westgate PM, Fleenor BS. Responsiveness to curcumin intervention is associated with reduced aortic stiffness in young, obese men with higher initial stiffness. $J$ Funct Foods. 2017;29:154-160. doi:10.1016/j.jff.2016.12.013
44. Oliver JM, Stoner L, Rowlands DS, et al. Novel form of curcumin improves endothelial function in young, healthy individuals: a double-blind placebo controlled study. $J$ Nutr Metab. 2016;2016:1089653. doi:10.1155/2016/1089653

45. Akazawa N, Choi Y, Miyaki A, et al. Curcumin ingestion and exercise training improve vascular endothelial function in postmenopausal women. Nutr Res. 2012;32:795-799. doi:10.1016/j.nutres.2012.09.002

46. Yao Y, Wang W, Li M, Ren H, Chen C. Curcumin exerts its antihypertensive effect by down- regulating the AT 1 receptor in vascular smooth muscle cells. Sci Rep. 2016;6:25579. doi:10.1038/srep25579

47. Cas MD, Ghidoni R. Dietary curcumin: correlation between bioavailability and health potential. Nutrients. 2019;11:1-14.

48. Brosnan MJ, Devlin AM, Clark JS, Mullins JJ, Dominiczak AF. Different effects of antihypertensive agents on cardiac and vascular hypertrophy in the transgenic rat line TGR(mRen2)27. Am J Hypertens. 1999;12:724-731. doi:10.1016/S0895-7061(99)00021-7

49. Rakušan D, Kujal P, Kramer HJ, et al. Persistent antihypertensive effect of aliskiren is accompanied by reduced proteinuria and normalization of glomerular area in Ren-2 transgenic rats. Am J Physiol. 2010;299:758-766. doi:10.1152/ajprenal.00259.2010

50. Giraudeau C, Geffroy F, Mériaux S, et al. 19F molecular MR imaging for detection of brain tumor angiogenesis: in vivo validation using targeted PFOB nanoparticles. Angiogenesis. 2013;16:171-179. doi:10.1007/s10456-012-9310-0

51. Ellena JF, Obraztsov VV, Cumbea VL, Woods CM, Cafiso DS. Perfluorooctyl bromide has limited membrane solubility and is located at the bilayer center. Locating small molecules in lipid bilayers through paramagnetic enhancements of NMR relaxation. J Med Chem. 2002;45:5534-5542. doi:10.1021/jm020278x

52. Rother S, Galiazzo VD, Kilian D, et al. Hyaluronan/collagen hydrogels with sulfated hyaluronan for improved repair of vascularized tissue tune the binding of proteins and promote endothelial cell growth. Macromol Biosci. 2017;17:1-13. doi:10.1002/mabi.201700154

53. Dogné S, Flamion B. Endothelial glycocalyx impairment in disease focus on hyaluronan shedding. Am J Pathol. 2020;190:768-780. doi:10.1016/j.ajpath.2019.11.016

54. Beldman TJ, Senders ML, Alaarg A, et al. Hyaluronan nanoparticles selectively target plaque-associated macrophages and improve plaque stability in atherosclerosis. ACS Nano. 2017;11:5785-5799. doi:10.1021/acsnano.7b01385

55. Hou X, Lin H, Zhou X, et al. Novel dual ROS-sensitive and CD44 receptor targeting nanomicelles based on oligomeric hyaluronic acid for the efficient therapy of atherosclerosis. Carbohydr Polym. 2020;232:1-8. doi:10.1016/j.carbpol.2019.115787

56. Boarescu PM, Boarescu I, Bocșan IC, et al. Curcumin nanoparticles protect against isoproterenol induced myocardial infarction by alleviating myocardial tissue oxidative stress, electrocardiogram, and biological changes. Molecules. 2019;24(15):2802. doi:10.3390/ molecules24152802

57. Kilinç E, Kaygisiz Z, Benek BS, Gümüştekin K. Effects and mechanisms of curcumin on the hemodynamic variablesof isolated perfused rat hearts. Turk J Med Sci. 2016;46(1):166-173. doi:10.3906/sag-1410-131

58. Marczylo TH, Steward WP, Gescher AJ. Rapid analysis of curcumin and curcumin metabolites in rat biomatrices using a novel ultraperformance liquid chromatography (UPLC) method. J Agric Food Chem. 2009;57(3):797-803. doi:10.1021/jf803038f 


\section{Publish your work in this journal}

The International Journal of Nanomedicine is an international, peerreviewed journal focusing on the application of nanotechnology in diagnostics, therapeutics, and drug delivery systems throughout the biomedical field. This journal is indexed on PubMed Central, MedLine, CAS, SciSearch ${ }^{\mathbb{B}}$, Current Contents ${ }^{\mathbb{B}} /$ Clinical Medicine, $^{2}$
Journal Citation Reports/Science Edition, EMBase, Scopus and the Elsevier Bibliographic databases. The manuscript management system is completely online and includes a very quick and fair peer-review system, which is all easy to use. Visit http://www.dovepress.com/ testimonials.php to read real quotes from published authors. 